Einführung zum Thema

Anaesthesist 2020 69:857-859

https://doi.org/10.1007/s00101-020-00834-2

(c) Springer Medizin Verlag GmbH, ein Teil von Springer Nature 2020
A. R. Sauter' $\cdot$ M. M. Luedi ${ }^{2}$

'Abteilung für Anästhesiologie, Oslo Universitätskrankenhaus - Rikshospitalet, Oslo, Norwegen

${ }^{2}$ Universitätsklinik für Anästhesiologie und Schmerztherapie, Inselspital, Universität Bern, Bern, Schweiz

\title{
Effektive Schmerzbehandlung durch Blockadetechniken im Rahmen multimodaler Therapie
}

In der aktuellen Ausgabe von Der Anaesthesist stellen Steinfeldt et al. eine Übersichtsarbeit $\mathrm{zu}$ ultraschallgeleiteten peripheren Rumpfwandblockaden vor [1]. Die Arbeit gibt eine sehrgute Zusammenfassung der relevanten Blockadetechniken und ist dank ihrer anschaulichen Bilder und Beschreibungen ein geeignetes Hilfsmittel für den klinischen Alltag. Zugleich erhält der Leser eine kritische Bewertung der Evidenzlage der vorgestellten Techniken.

Viele Patienten, die sich einem abdominalen und/oder thorakalen chirurgischen Eingriff unterziehen müssen, erleben postoperativ moderate oder starke Schmerzen [2]. Eine unzureichende postoperative Schmerzbehandlung kann zu verzögerter Rehabilitation und verlängertem Krankenhausaufenthalt des Patienten führen. Bei etwa $10 \%$ der Patienten gehen akute und schwer zu kontrollierende postoperative Schmerzen in einen persistierenden chronischen Schmerzzustand über [3]. Der Gebrauch von systemisch wirkenden Opioiden muss wegen des Auftretens von Nebenwirkungen wie Übelkeit, Erbrechen und Juckreiz kritisch gesehen werden. Opiatabhängigkeit erreichte in den USA in den vergangenen Jahren epidemische Dimensionen [4]. Zudem wird das Auftreten einer opioidinduzierten Hyperalgesie für die Entwicklung persistierender postoperativer Schmerzen mitverantwortlich gemacht [3]. Der Einsatz von neuroaxialen Blockaden stellt zwar eine willkommene und wirksame Alternative zur systemischen Schmerzbehandlung dar, aber Nebenwirkungen wie Blutdruckfall, Harnverhalt und reduzierte Mobilität sowie ein relativ hohes Risiko für schwerwiegende Komplikationen wie peridurale Blutungen und Infektionen begrenzen den Einsatz der neuroaxialen Schmerzverfahren [3].

\section{》) Schmerzintensität und Opioidverbrauch lassen sich mithilfe peripherer Rumpfwand- blockaden reduzieren}

Bei vielen chirurgischen Eingriffen ist die Verwendung peripherer Nervenblockaden eine wichtige Alternative oder Ergänzung für die perioperative Schmerzbehandlung. Klassische periphere Nervenblockaden im Bereich der Extremitäten sind meist auf spezifische Nerven gerichtet und führen zur vollständigen Anästhesie und Analgesie im entsprechenden Innervationsgebiet $[5,6]$. Rumpfwandblockaden sind dagegen in ihrer Wirkung oft weniger klar abgegrenzt und für eine viszerale Analgesie ungeeignet. Rumpfwandblockaden sollten daher v.a. im Rahmen einer multimodalen Schmerzbehandlung verwendet werden [1]. Postoperativ können bei der Verwendung peripherer Rumpfwandblockaden eine reduzierte postoperative Schmerzintensität und ein verminderter Opioidverbrauch nachgewiesen werden [7-9].

Bei den meisten Rumpfwandblockaden wird das Lokalanästhetikum nicht unmittelbar in die Nähe eines peripheren Nervs injiziert. Bei den sog. interfaszialen Blockaden wird die Injektionsnadel vielmehr in einen Interfaszialraum platziert [10]. Durch Verabreichung eines relativen hohen Injektionsvolumens erreicht das Lokalanästhetikum dann die Zielnerven.

Einige der Rumpfwandblockaden wie z.B. der „Transversus-abdominisplane“(TAP)-Block wurden anfänglich als landmarkengesteuerte Methoden durchgeführt [11]; heute werden meist ultraschallgeleitete Verfahren eingesetzt. Im Vergleich zu den neuroaxialen Blockaden sind viele der ultraschallgeleiteten Blockaden technisch einfacher durchzuführen. Aufgrund einer größeren Entfernung der Injektionsnadel zu anatomischen Risikostrukturen können zudem Komplikationen wie Blutungen im Periduralraum oder ein Pneumothorax vermieden werden. Da auch ein direkter Kontakt zwischen Nadel und peripheren Zielnerven bei den meisten interfaszialen Blockaden vermieden wird, ist das Risiko für traumatische Nervenschäden stark reduziert. Die für periphere Extremitätenblockaden verbreitete zusätzliche Verwendung von elektrischer Nervenstimulation zum Ultraschall, die „dual guidance“, ist daher nicht erforderlich [12].

Allerdings ist die geringe Präzision der Nadelposition im Verhältnis zu den Zielnerven auch eine wesentliche Beschränkung vieler Rumpfwandblockaden, die auf dem Prinzip einer interfaszialen Injektion basieren. Ein erfolgreicher Block ist von einer günstigen, aber schließlich zufälligen Ausbreitung des Lokalanästhetikums abhängig. Lönnqvist und Karmakar vergleichen dieses Prinzip mit dem Gebrauch einer Schrotflinte, die in der Hoffnung verwendet wird, dass min- 
destens eines der Pellets die Zielscheibe trifft [13]. Die Verwendung großer Injektionsvolumina von Lokalbetäubungsmitteln, die bei den meisten Faszienblockaden erforderlich sind, ist mit dem Risiko für eine systemische Toxizität verbunden [14]. Bei bilateralen Blockaden ist das Risiko einer raschen Absorption des Lokalbetäubungsmittels mit hoher Plasmakonzentration besonders hoch.

Mit den meisten interfaszialen Rumpfwandblockaden kann eine oberflächliche somatische Analgesie erreicht werden [15]. Ein viszeraler Effekt, der über eine Ausbreitung des Lokalanästhetikums zu den Spinalnerven und zum sympathischen Grenzstrang im thorakalen paravertebralen Raum erreicht werden kann, wird bei einigen Blockadetechniken diskutiert. So legen anatomische Studien am Kadaver eine paravertebrale Ausbreitung des Lokalbetäubungsmittels bei der anterioren Quadratus-lumborumBlockade und bei der „Erector-spinaeplane"-Blockade nahe $[16,17]$. Die Effektivität einer Injektion in weitem Abstand zum thorakalen paravertebralen Raum sollte trotzdem kritisch hinterfragt werden [13]. Mit einer ultraschallgeleiteten paravertebralen Blockade, wie in der Beschreibung der peripheren Rumpfwandblockaden von Steinfeldt et al. dargestellt, kann eine zuverlässigere viszerale Anästhesie erreicht werden als mit den beschriebenen interfaszialen Blockaden [1].

Andere bisher wenig beachtete Eigenschaften der Körperfaszien werden für den Effekt der interfaszialen Rumpfwandblockaden diskutiert. So vermuten Blanco et al., dass ein dichtes Netzwerk sympathischer Nervenfasern innerhalb der Fascia thoracolumbalis den analgetischen Effekt der von ihnen beschriebenen posterioren Quadratus-lumborum-Blockade erklären kann [18]. Bei den oft hohen Injektionsvolumina kann unter Inkaufnahme eines nichtauszuschlieBenden Risikos von toxischen Nebenwirkungen auch mit einem systemischen analgetischen Effekt des Lokalanästhetikums gerechnet werden. Die perioperative analgetische Wirkung durch Lidocaininfusion wurde in zahlreichen Studien belegt [17].
Trotz des wachsenden Interesses an den Rumpfwandblockaden gibt es in anästhesiologischen Kreisen erstaunlicherweise nur begrenzte Erfahrung mit den beschriebenen Techniken. In einer landesweiten Umfrage, die 2018 unter den Mitgliedern der Schweizerischen Gesellschaft für Anästhesiologie und Reanimation durchgeführt wurde, hatten beispielsweise lediglich $60 \%$ der befragten Anästhesisten Erfahrung mit TAP-Blockaden [12]. Bei Quadratuslumborum und Erector-spinae-planeBlockaden waren es sogar nur $6 \%$ resp. $1 \%$ der befragten Kollegen. Es hatten $29 \%$ der Teilnehmer an der landesweiten Umfrage klinische Erfahrung mit paravertebralen Blockaden [12].

Die Übersichtsarbeit von Steinfeld et al. leistet einen wichtigen Beitrag zu Verständnis und angeleitetem Erlernen ultraschallgeleiteter peripherer Rumpfwandblockaden [1]. Die meisten der im Beitrag vorgestellten Blockadetechniken eignen sich zur effektiven Schmerzbehandlung im Rahmen einer multimodalen Therapie. Die Wirkungsmechanismen, die interfaszialen Verbreitungswege des Lokalbetäubungsmittels und die daraus hervorgehenden Indikationen der Blockadetechniken müssen jedoch in zukünftigen Untersuchungen weiter erforscht werden.

\section{Korrespondenzadresse}

\section{Dr. A. R. Sauter, PhD}

Abteilung für Anästhesiologie, Oslo

Universitätskrankenhaus - Rikshospitalet

Oslo, Norwegen

sauter@start.no

Interessenkonflikt. A.R. Sauter und M.M. Luedi geben an, dass kein Interessenkonflikt besteht.

\section{Literatur}

1. Steinfeldt $T$, Kessler $P$, Vincent $O$, Lang $P$, Schwemmer U, Döffert J, Mathioudakis D, Hüttemann E, Armbruster W, Sujatta S, Lange M, Weber S, Reisig F, Hillmann R, Volk T, Wiesmann T (2020) Periphere Rumpfwandblockaden - Übersicht und Bewertung. Anaesthesist. https://doi.org/10. 1007/s00101-020-00809-3.

2. Gerbershagen HJ, Pogatzki-Zahn E, Aduckathil S et al (2014) Procedure-specific risk factor analysis for the development of severe postoperative pain. Anesthesiology 120:1237-1245
3. Glare P, Aubrey KR, Myles PS (2019) Transition from acute to chronic pain after surgery. Lancet 393:1537-1546

4. Kent ML, Hurley RW, Oderda GM et al (2019) American Society for Enhanced Recovery and Perioperative quality initiative- 4 joint consensus statement on persistent postoperative opioid use: definition, incidence, risk factors, and health care system initiatives. Anesth Analg 129:543-552

5. Enneking FK, Chan V, Greger J et al (2005) Lowerextremity peripheral nerve blockade: essentials of our current understanding. Reg Anesth Pain Med 30:4-35

6. Klaastad O, Sauter AR, Dodgson MS (2009) Brachial plexus block with or without ultrasound guidance. Curr Opin Anaesthesiol 22:655-660

7. Krohg A, Ullensvang $K$, Rosseland LA et al (2018) The analgesic effect of ultrasoundguided quadratus lumborum block after cesarean delivery: a randomized clinical trial. Anesth Analg 126:559-565

8. Abdallah FW, Chan VW, Brull R (2012) Transversus abdominis plane block: a systematic review. Reg Anesth Pain Med 37:193-209

9. Versyck B, Van Geffen GJ, Chin KJ (2019) Analgesic efficacy of the Pecs Il block: a systematic review and meta-analysis. Anaesthesia 74:663-673

10. Elsharkawy H, Pawa A, Mariano ER (2018) Interfascial plane blocks: back to basics. Reg Anesth Pain Med 43:341-346

11. Mcdonnell JG, O'donnell BD, Farrell T et al (2007) Transversus abdominis plane block: a cadaveric and radiological evaluation. Reg Anesth Pain Med 32:399-404

12. Luedi MM, Upadek V, Vogt AP et al (2019) A Swiss nationwide survey shows that dual guidance is the preferred approach for peripheral nerve blocks. Sci Rep 9:9178

13. Lönnqvist PA, Karmakar M (2019) Close-to-thenerve vs interfascial plane blocks: Sniper rifle vs shotgun-which will hit the target most reliably? Acta Anaesthesiol Scand 63:1126-1128

14. Rahiri J, Tuhoe J, Svirskis D et al (2017) Systematic review of the systemic concentrations of local anaesthetic after transversus abdominis plane block and rectus sheath block. Br J Anaesth 118:517-526

15. Chin KJ, Mcdonnell JG, Carvalho B et al (2017) Essentials of our current understanding: abdominal wall blocks. Reg Anesth Pain Med 42:133-183

16. Dam M, Moriggl B, Hansen CK et al (2017) The pathway of Injectate spread with the transmuscular quadratus lumborum block: a cadaver study. Anesth Analg 125:303-312

17. Vidal E, Giménez H, Forero M et al (2018) Erector spinae plane block: a cadaver study to determine its mechanism of action. Rev Esp Anestesiol Reanim 65:514-519

18. Blanco R, Ansari T, Riad W et al (2016) Quadratus Lumborum block versus transversus abdominis plane block for postoperative pain after cesarean delivery: a randomized controlled trial. Reg Anesth Pain Med 41:757-762 
Hier steht eine Anzeige.

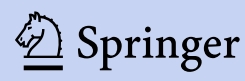

